\title{
Les « Roms migrants » en France \\ Du fait migratoire à la construction d'un problème public
}

\author{
Samuel Delépine \\ Maître de conférences en géographie. Université d'Angers. UMR ESO.
}

La migration de quelques 20000 roms roumains, bulgares ou des Balkans vers la France fait beaucoup parler au niveau politique comme dans les médias. Pourtant ce phénomène migratoire est sans dimension numérique majeure mais il semble être devenu un enjeu politique incontournable. Les racines de ce qui est ici récupéré et présenté comme un problème public sont lointaines et associées à des représentations sociales fortement marquées depuis des siècles en Europe à l'égard des Roms, des Tsiganes ou des Gens du Voyage sans que le public sache de qui l'on parle exactement. La confusion et les amalgames sont légions et les craintes qui y sont associées également. Les processus de relégation des Tsiganes sont historiques et il ne faut pas voir dans la stigmatisation de quelques migrants un simple «coup» politique. En effet, ce qui ce passe actuellement, aussi bien dans les pays d'émigration que dans ceux d'immigration (nous prendrons l'exemple de la France) est révélateur du nouvel avènement d'une question rom qui se serait appelée «tsigane » ou «nomade» autrefois et dont les mécanismes inquiétants se doivent d'être révélés et déconstruits. 


\section{Tsiganes, Roms, « Roms migrants » : de qui parle-t-on ?}

\section{Roms et/ou Tsiganes : l'obsession de définir un peuple et de connaître son origine}

Mais qui sont-ils ? D'où viennent-ils...Il semble qu'au-delà de la construction d'un problème public autour d'un fait migratoire peu important que nous détaillerons ci-après, savoir qui sont les Roms soit la principale autre question qui se pose à ceux qui s'y intéressent notamment politiques et médias.

Ce texte n'a pas vocation à répondre à cette question de l'origine et de la définition « peuple tsigane ». Bien au contraire. Parce qu'il apparait assez clairement que l'exclusion sociale dont sont l'objet une grande partie de ceux que l'on appelle « Roms » aujourd'hui en Europe vient justement de cette volonté séculaire de les catégoriser, de les identifier, de les nommer et d'en faire un peuple uni voire une nation au mépris de la diversité des familles, des individus et des situations qu'ils vivent sur les territoires. Ainsi, sous prétexte que la langue romani contient des mots hérités du sanskrit, l'origine indienne semble attestée et tous les Roms viendraient d'Inde qu'ils auraient quitté il y a environ mille ans sans que personne ne sache pourquoi. La thèse de razzias effectués par l'Empire Seldjoukide de la Turquie actuelle jusqu'au bord du Gange en créant une catégorie à part de sous-citoyens est beaucoup plus crédible (Clanet, 2011). Il n'est ainsi pas surprenant de retrouver dans cette langue, qui varie d'un groupe à l'autre, d'un village à l'autre, des influences persanes : «j'ai été très surpris en discutant avec un iranien à Londres, je comprenais des mots de sa langue, notamment les chiffres qui étaient presque pareils ». ${ }^{1}$

Cet exemple montre que dès l'origine qui obsède tant d'historien, de politiques ou de militants roms eux-mêmes, les « Tsiganes » constituaient déjà un groupe très mélangé et contraint.

Les Tsiganes sont arrivés, on pourrait dire qu'ils ont été amenés, sur le sol européens au XVème siècle et se sont dispersés, au gré des contraintes (500 ans d'esclavage en Roumanie, asservissement en Autriche-Hongrie...) sur tout le continent, se mélangeant et constituant des groupes distincts en Europe dont les principaux sont: les Roms en Europe centrale et orientale, les Gitans dans la péninsule ibérique et les Sinti et Sinti-Manus en Europe

\footnotetext{
${ }^{1}$ Entretien auprès d'un jeune rom migrant de retour d’Angleterre. Réalisé en Roumanie. Décembre 2014.
} 
occidentale et du nord. Ce ne sont là que trois très grands groupes eux-mêmes subdivisés en multiples sous-groupes.

Le terme générique fut longtemps «Tsiganes » pour les qualifier tous malgré les différences locales et régionales. Ce sont les Zigueuner (Tsiganes) ainsi catégorisés et sans distinction que les nazis ont voulu exterminer pendant la seconde guerre mondiale en les déportant dans tous les camps de la mort dont celui d'Auschwitz où le « camp des familles » leur était réservés. Aujourd'hui le mot «rom »s'est substitué à «tsigane » dans un processus qui a démarré après la chute du mur de Berlin. Les institutions européennes en lien avec certaines ONG roms très actives militent pour l'emploi de ce terme et c'est ainsi que dans cette approche globale, le gitan andalou, le Manus breton et même le traveller irlandais qui dans la majorité des cas n'a même pas d'origine balkanique (indienne n'en parlons pas) sont tous devenus : des Roms.

On comprend que dans cette logique globalisante la théorie de l'origine commune soit soutenue et diffusé (c'est elle que met en évidence le portail du site web de l'Union Européenne par exemple) car elle nécessaire à l'avènement d'un projet politique global qui concerne une population au sens large. Mais elle pose un réel problème de contournement des réalités historiques, géographiques, identitaires, économiques et sociales des premiers concernés. En tant que chercheur et même lorsqu'on veut rétablir certaines vérités on ne peut donc ignorer la question des origines. Non pas pour savoir «qui sont les Roms » mais parce qu'elle partie prenante d'un projet politique global (Delépine, 2012) qui influence directement les premiers concernées et plus encore quand une partie d'entre eux est en situation de migration.

\section{Qui appelle-t-on les Roms migrants ?}

Pour éviter les confusions avec les Gens du Voyage (catégorie administrative francofrançaise) ou les Tsiganes en général, l'appellation "Roms migrants » s'est démocratisée depuis une dizaine d'année en France et en Europe («Roma migrants »). Sont ainsi désignés les Roms qui migrent, surtout depuis les années 2000 même si le phénomène a débuté dès 1990, depuis 1'Est de 1'Europe vers l'Europe occidentale. En France, ce sont environ 20000 personnes, essentiellement roumaines et bulgares, dont nous parlons ici. Soit un poids numérique et démographique très faible dans un pays de 65 millions d'habitants. 
Cette appellation de « roms migrants » peut créer des confusions au sein d'une thématique qui souffre déjà de confusions et d'amalgames multiples entre les différents noms de groupes tsiganes. En effet, le mot migrant peut troubler dans un contexte général de méconnaissance de ces populations et de perceptions fausses et historiques sur les nomades, les Tsiganes, les Roms... Ainsi, l'appellation «Roms migrants » peut renvoyer au nomadisme ou à l'errance ce qui n'a pas lieu d'être pour qui connaît le sujet mais « en dehors des cercles universitaires, la catégorie ethno-juridique de "nomade » n'est toujours pas réellement remise en question » (Cousin,2009). Il convient de savoir que les environs huit millions de Roms, ou catégorisés comme tels, vivant en Europe centrale et orientale sont sédentaires et que sur les dix à douze millions de ceux qui sont appelés Tsiganes en Europe 90\% sont sédentaires.

Ces personnes sont avant tout des migrants roumains, bulgares, kosovars...mais ce n'est pas ainsi qu'ils sont considérés. L'inconscient collectif insiste sur l'ethnie, le rom ou le tsigane, et le classe dans une catégorie de nomades instables, ingérables et criminalisés (Olivera, 2008).

\section{Un flux migratoire peu important vers la France mais aux forts impacts}

\section{De forts ancrages territoriaux contrairement aux stéréotypes}

La situation des Roms en Europe centrale et orientale s'est révélée depuis la chute du mur à certains experts et depuis quelques années, via la migration est-ouest fortement médiatisée, à l'ensemble de l'Europe occidentale. Les situations les plus misérables dans des ghettos ou des bidonvilles ont interpellé du chercheur au journaliste et ont été fortement médiatisées. Elles masquent complètement la diversité des groupes, des familles et des individus et favorise aujourd'hui la constitution d'un groupe, qui serait homogène du point de vue ethnique et social et que l'on pourrait résumer aux « Roms », pauvres et misérables exclus de l'Europe ». Les Roms sont sédentaires depuis des siècles en Europe centrale et orientale et les plus mobiles, ceux qui ont la possibilité de migrer, sont ceux qui ont les moyens de cette mobilité. C'est une réalité souvent ignorée car les occidentaux ont le sentiment de voir arriver les plus misérables, et donc inquiétants, d'entre eux. Les plus pauvres se maintiennent dans leurs 
quartiers ou dans leurs villages d'origine soit parce qu'ils ont toujours été astreints à certaines tâches sans possibilités de développement soit parce que leur activité économique s'organise sur un espace assez réduit et proche du lieu d'habitation. Il faut noter à ce sujet que les périodes douloureuses de l'histoire des Roms (esclavage en Valachie et en Roumanie, fixation forcée sous les Habsbourg ou pendant la période communiste) ont contribué à ce que Roms et non roms « acceptent » l'idée que certains espaces urbains ou ruraux sont justement ceux des Roms et qu'à l'inverse d'autres ne le sont pas et qu'ils n'ont rien à faire comme les centresvilles ou ce qu'on appelle les beaux quartiers.. Seuls les plus riches rompent ces ségrégations sociospatiales historiques pour investir ces espaces privilégiés, souvent de façon très ostentatoire, au risque d'être qualifiés d'envahisseurs. Malgré une dispersion plus complexe et souvent plus individuelle sur l'espace, presque toutes les villes d'Europe centrale et orientale ont ainsi leur «quartier tsigane » au sein duquel la population résidente est en général très peu mobile ${ }^{2}$.

Des processus ségrégatifs existent et touchent ces « espaces tsiganes ou roms » mais ils ont des degrés variables et n'excluent pas toujours un sentiment d'attachement ou d'appropriation du territoire. Ces facteurs de stabilité sur un espace considéré comme sien donnent à la population rom des pays d'Europe centrale un enracinement parfois séculaire qui peut toutefois être remis en cause à tout moment et pour diverses raisons qui peuvent être par exemple des expulsions liées à un projet immobilier ou commercial, une trop forte ségrégation ou un repli communautaire et un fort entre soi. Ce rapport au territoire compris ici comme «le marquage spatial de la conscience historique d'être ensemble ${ }^{3} \mathrm{n}$ 'a a priori rien d'une caractéristique «tsigane». Seuls les phénomènes de ségrégations extrêmes ou à l'inverse d'entre soi communautaire constituent des variables liées à la «tsiganité » ou la « romanité » des lieux. Ces évènements qui provoquent des changements visibles ne sont pas très nombreux mais ils restent les plus remarquables et les plus médiatisés.

\section{Une mobilité quasi nulle}

Le sociologue Jean-Pierre Liégeois parle de «potentiel » de mobilité ${ }^{4}$ pour requalifier ce que d'aucun affirme être un nomadisme naturel au sujet des Roms. Pour beaucoup de

\footnotetext{
${ }^{2}$ Samuel Delépine, Quartiers Tsiganes. L'habitat et le logement des Rroms de Roumanie en question, L'Harmattan, Paris, 2007.

${ }^{3}$ Alain Tarrius, Les nouveaux cosmopolitismes. Mobilités, identités, territoires, Ed de l'Aube, 2000.

${ }^{4}$ Jean-Pierre Liégeois, Roms en Europe, Editions du Conseil de l'Europe, 2007.
} 
familles ce «potentiel»n'a rien à voir avec le fait d'être tsigane, ou indirectement si la migration est due à des discriminations. Pour beaucoup d'ailleurs il n'existe même pas quand bien même la capacité à être mobile serait une caractéristique, là aussi très fantasmée, d'une «culture tsigane » que nous nous garderons bien de définir ou de justifier. Ainsi les familles roms qui en ont la capacité savent très bien quels types de territoires devront être investis en cas de grave crise ou de contraintes trop fortes. C'est ce qui se produit depuis la chute du mur et plus encore depuis 2007 avec l'émergence de nouvelles mobilités internationales vers des territoires choisis à l'origine pour leurs caractéristiques locales et a priori favorables du point de vue économique.

C'est une migration tout à fait classique en somme et qu'il faut mesurer et pondérer. Tout d'abord, sans même parler de migration internationale, rappelons que la mobilité interne des Roms en Europe centrale et orientale est tout à fait réduite. Elle dépend principalement du statut social de l'individu ou de la famille et de ce point de vue, beaucoup de Roms étant en situation précaire dans des quartiers souvent relégués, leur mobilité est quasi nulle.

Ceux qui entreprennent une migration vers l'Europe occidentale et qui s'en donnent les moyens le font, comme tout migrant, avec un projet migratoire bien pensé (économique, santé, scolarisation). Nous sommes très loin d'une horde de nomades potentiels, de millions d'individus prêts à envahir l'Europe Occidentale parce que telle serait leur culture, celle du déplacement et de l'investissement opportunistes de territoires. Ceci n'est que fantasme. Evoquer le destin des familles roms d'Europe centrale et orientale c'est d'abord rappeler le maintien de la quasi-totalité d'entre elles sur leur territoire d'origine.

\section{Une « migration rom » spécifique?}

Nous venons de décrire un phénomène migratoire peu important et assez classique, avec des migrants économiques, mais ce mouvement n'a-t-il pas quelques spécificités ?

Si l'on prend l'exemple de la Roumanie, le mouvement migratoire des Roms de ce pays n'a rien d'exceptionnel. Il s'agit donc avant tout de migrants économiques avec des projets qui diffèrent et ceci malgré l'apparente homogénéité, visuelle notamment, des groupes et des familles. Comme nous l'avons dit le flux migratoire n'est pas exceptionnel non plus du point de vue numérique puisqu'au sein des Roumains qui migrent vers l'Ouest, les Roms sont en proportion égale à ce qu'ils représentent dans la population totale du pays soit environ 
10\%. Une partie des Roms invoque la fuite des discriminations mais force est de constater que ce sont principalement les raisons économiques qui guident ces migrants soucieux de démontrer leur réussite au niveau local si toutefois un projet de retour existe. La réussite de quelques uns motive les autres et c'est ainsi que des zones d'émigration se forment. Des villages, des quartiers entiers se vident quand d'autres espaces pourtant similaires ne subissent pas ce phénomène.

La circulation sur des territoires choisis n'est pas qu'opportunité conjoncturelle même si l'évolution immédiate des politiques locales dirige évidemment les flux. Il faut inscrire ce mouvement dans la logique structurelle des migrations internationales pour dépasser les fantasmes et les clichés.

Alors qu'aurait-elle de «rom » ou de «tsigane » cette migration ? Elle est familiale tout d'abord ce qui peut rompre avec l'image classique du migrant associée à l'homme seul et plutôt jeune. Elle peut même concerner des familles élargies et voir, comme nous le disions précédemment, les habitants d'un quartier, ou partie de celui-ci se déplacer au gré d'une opportunité d'un accueil favorable sur un territoire donné. Dans la plupart des villes françaises, de grandes métropoles en général, où des familles roms se sont installées, elles sont presque toutes de la même ville ou au moins du même département.

C'est aussi une migration d'une certaine homogénéité sociale. En effet, bien que les projets migratoires varient en termes de priorité d'une famille à l'autre (scolarisation des enfants, emploi, soins, activités pendulaires licites ou non...) la plupart des individus sont au bas de l'échelle sociale et peu qualifiés ce qui ne facilite pas leur insertion directe. Cela les contraint même beaucoup. Ciblés par les politiques sécuritaires en France notamment, leur manque de ressources et d'inscription sur le marché du travail, certes ouvert depuis le $1^{\text {er }}$ janvier 2014 aux Roumains et aux Bulgares, en font des cibles idéales pour les expulsions du territoire.

Enfin, et c'est issu de cette apparente homogénéité sociale, leur installation dans des interstices de l'espaces urbains, sur des franges improbables, en zone de regroupement pour former des bidonvilles semble aussi les caractériser. Ces «campements illicites » pour reprendre l'expression quasi officielle puisque parler de bidonvilles semble délicat et proscrit aujourd'hui en France ne sont pourtant pas une nouveauté. D'autres migrants en ont été réduits à ce type d'installation mais aujourd'hui ces bidonvilles caractérisent les Roms et ils sont d'abord identifiés par cela et par certaines de leurs activités visibles comme la mendicité ou comme les activités illicites de certains mafieux (prostitution) sans que les projets des migrants, par famille, soient pris en compte et ait une quelconque chance d'aboutir. 
Ces deux derniers points, fortement instrumentalisés, donne une image très négative de ces migrants et sont associés aux stéréotypes historiques d'un «peuple» différent, inquiétant et ayant un mode de vie incompatible, notamment parce que très mobile par nature (ce que nous venons de déconstruire) avec le mode de vie majoritaire.

\section{Intégration, accès au droit et intervention sociale : quand la logique du problème public ethnicisé prend le dessus}

\section{Bidonvilles et squattes}

La majorité des Roms migrants en France, et c'est également le cas dans les autres pays d'Europe occidentale, vit dans des bidonvilles ou des squattes. Cela les rend visible et c'est une réalité assez insupportable pour les autochtones que de voir ces migrants qu'ils jugent inquiétant et instables dans une misère ostentatoire. D'autant plus inquiétant, a priori, que ces derniers n'ont effectué que 2000 kilomètres tout au plus avant de s'installer dans des interstices de l'espace urbain qu'ils jugent accessibles.

L'occupation illégale des terrains et le refus d'une prise en charge locale durable contraignent les familles à des déplacements multiples, au gré des expulsions des terrains qu'ils occupent par les forces de police et ordonnées par les élus locaux et par les préfectures. Ceci renforce le cliché du tsigane nomade or le déplacement est contraint. Ici, chaque expulsion oblige à un nouveau déplacement, parfois une famille ou un groupe peut-être expulsé quinze fois en une année (Delépine,2012,p85). La scolarisation est considérée comme le premier facteur d'intégration durable pour les plus jeunes. Aujourd'hui en France, la scolarisation des enfants roms migrants dépend de la coordination de nombreux acteurs dont les familles elles-mêmes. Il est un fait que la scolarisation dès l'école primaire augmente les chances du jeune, les exemples d'une insertion réussie à l'école sont nombreux et relevés comme bonnes pratiques ou modèles de réussite par les associations ou collectifs qui soutiennent les familles roms migrantes. Toutefois les expulsions récurrentes, les interruptions contraintes de la scolarité dans l'attente de trouver une nouvelle école ne permettent pas de franchir ce premier pas vers l'intégration pour de nombreux enfants et pour leurs familles. 
On reproche souvent aux Roms leur absence de volonté d'intégration et il faut d'abord, à ce sujet, identifier les projets migratoires de chacun. Certains n'ont effectivement pas cet objectif s'inscrivant dans des migrations pendulaires très fréquentes. Pour les autres, les conditions de l'intégration, notamment à cause de ces multiples expulsions que nous venons de décrire, ne sont pas réunies et leur reprocher sous prétexte de mode de vie ou de culture n'est qu'une échappatoire politique grossière mais qui globalement suffit à convaincre l'opinion publique.

\section{Des pressions nationales qui pèsent au niveau local}

Les directives de la circulaire du 26 août 2012 de la DIHAL (Délégation Interministérielle à l'Habitat et au logement) et consacrées aux campements dits illicites vont dans le sens d'un accompagnement des Roms, de leur accès aux droits et a priori, à terme, de leur intégration. Bien que cette circulaire ne soit pas explicitement destinée aux Roms pour des raisons républicaines de non ciblage d'un groupe ethnique particulier, il est clair que c'est d'eux dont il s'agit dans ce texte. Le texte pose d'emblée un problème important: il contraint à la « sélection » des familles après une obligation de diagnostic qui génère de fait une sorte de classement des intégrables et des autres. Il a néanmoins le mérite d'indiquer qu'il faut veiller "à la continuité de l'accès aux droits des personnes ${ }^{5}$ et de se pencher sur la scolarisation, l'emploi, la santé et le logement soit tous les domaines qui nécessitent une inscription dans le droit commun.

Aujourd'hui les résultats de cette circulaire d'abord adressée aux préfets de départements sont mitigés comme le soulignent de nombreuses associations sur le terrain et notamment parce que les préfets restent libre d'agir selon leur souhait face à ces campements et à la population qui y réside. Souvent, un cercle vicieux s'installe. Pas d'accès au logement ou à l'emploi sans tire de séjour et pas de titre de séjour sans emploi ou ouvertures de droits exceptionnels (logement, santé). Par exemple, la loi DALO (Droit Au Logement Opposable) s'applique aux ressortissants communautaires mais il faut pour cela un titre de séjour.

A cela s'ajoute le discours politique ambiant qui depuis la mise en place de cette circulaire n'a jamais facilité sa mise en œuvre au point qu'en 2015 les espoirs sont déçus. Le premier

\footnotetext{
${ }^{5}$ Voir l'ordonnance du 26 août 2012, ses recommandations et ses premiers effets sur http://www.gouvernement.fr/sites/default/files/fichiers/dihal_dpt__vademecum campements.pdfde. décembre 2013.
} 
ministre, alors ministre de 1'Intérieur parle d'absence de volonté d'insertion des Roms ${ }^{6}$ sans que les conditions de cette insertion soient réunies ou mises en œuvre.

$\mathrm{Au}$ contraire de pratiques inclusives, les procédures de contrôles, d'expulsions, de recours contre ces familles sont légion et permettent aussi de limiter leur accès aux droits. C'est la confusion entre la lutte pour les droits de l'Homme et contre les discriminations et la criminalisation ou le renvoie des Roms dans leur pays d'origine ${ }^{7}$. Les deux phénomènes évoluent en parallèle mais partent d'un socle culturaliste commun et de représentations de l'autre, ici du tsiganes, largement partagées par les populations majoritaires et globalement très négatives.

Les préfets ont également la main, sur l'insertion professionnelle. L'ouverture au marché du travail des ressortissants roumains et bulgares n'y changera presque rien et «il est tout à fait envisageable que les préfectures continuent à notifier des refus de séjour accompagnés d'une mesure d'éloignement ${ }^{8}$.

Le travail social et les travailleurs sociaux intervenants ou potentiellement intervenants auprès de ces familles se retrouvent prisonniers de cet état de fait car «confronté en permanence à des contradictions entre la commande publique, la mise en ouvre d'une éthique professionnelle et la nécessaire prise en compte de la réalité quotidienne des migrants » ${ }^{9}$. Le travailleur social doit se positionner. Entre respect de la commande, de la loi, de la personne, des bricolages pour son accès aux droits qui peuvent aller jusqu'à revendiquer le fait d'être un «délinquant solidaire », le travail social avec les migrants confronte droit et éthique. Avec les politiques nationales hostiles aux Roms, ces questions se posent directement aux travailleurs sociaux.

Les marges de manœuvres pour l'action en faveur de ces familles est donc très limitée même pour les professionnels de l'action sociale. Il n'y a pourtant qu'une réflexion vers l'accès au droit commun, dans le respect d'un cadre juridique certes contraignant, qui pourrait enclencher un processus d'intégration. Mais ce n'est globalement pas le sens de l'orientation politique actuelle au niveau national.

\footnotetext{
${ }^{6}$ «Roms : Manuel Valls affiche sa fermeté », Le Figaro, 14 mars 2013.

Manuel Valls : «Les Roms ont vocation à revenir en Roumanie ou en Bulgarie», Le Parisien, 24 septembre 2013

${ }^{7}$ Alain Reyniers, «La mobilité des Tsiganes en Europe : entre fantasmes et réalités », in Hermès, La revue, n 51 , 2008, CNRS Editions, P107 à111.

${ }^{8}$ «Fin de la période transitoire pour les ressortissants roumains et bulgares ». Note GISTI -Collectif Romeurope. Janvier 2014. P2.

${ }^{9}$ Brigitte Bouquet \& Marcel Jaeger, «Tensions entre mises en œuvre des politiques migratoires et questions éthiques du travail social », in : Hommes et Migrations, ${ }^{\circ} 1290,2011, \mathrm{p} 10-21$.
} 


\section{Des migrants dépendants des bonnes volontés locales?}

Dans ce contexte, il semble que l'insertion des familles roms migrantes ne puisse se faire que sur la base de bonnes volontés politiques, plutôt rares comme nous l'avons vu, relayées par le soutien d'associations ou divers collectifs. Beaucoup de projets émergent au niveau local indépendamment de la préfecture et de la politique d'Etat. Ils sont basés sur la volonté des familles, le soutien associatif et l'acceptation en dernier lieu de telle ou telle commune d'une agglomération, de tel ou tel élu, d'un employeur bienveillant...

D'autre part, les freins à ce processus que peuvent mettre en place les services de l'Etat nécessitent une vigilance accrue de la part des militants associatifs qui sont confrontés à des barrières politiques de plus en plus ethnicisées et qui ciblent directement les Roms. C'est une expérience que j'ai pu faire moi-même en préfecture en accompagnant un jeune rom pour son titre de séjour ${ }^{10}$.

Et les exemples ne manquent pas, localement, de stratégies pour le non accès aux droit des Roms. Cet «accès aux droits des Roms », rappelons-le, ne signifie rien sur le plan juridique puisque ces personnes devraient avant tout être considérées comme des nationaux. A l'échelle nationale également, les exemples de rejet ou de refus de l'accès aux droits sont nombreux. Citons la délivrance de prestations aux étrangers en situation régulière (2007) par les CAF et qui est un support mobilisé face aux familles roms pour la plupart en situation irrégulière. Celles qui avaient des droits ouverts avant 2007 s'en remettent aux Défenseur des Droits quand elles ont l'initiative d'un tel recours.

Alexandra Nacu relève que les démarches que peuvent entreprendre des Roms vers les services de soins et notamment vers les hôpitaux ou maternités se font dans des secteurs déjà défavorisés et surchargés par des situations sanitaires plus précaires ou plus graves que la moyenne (Nacu, 2009). En effet les espaces occupés par ces migrants sont en général aux marges périphériques les plus délaissées des agglomérations et donc très souvent les plus pauvres. L'oubli des territoires et de la géographie la plus élémentaire doit être signalé car il

\footnotetext{
${ }^{10}$ «On a des consignes sur les Roms ». Entendu le mercredi 26 juin 2013 au Bureau des Etrangers de la préfecture du Maine-et-Loire.
} 
nous rappelle que l'approche globale que propose «la question rom » ignore les territoires, les ancrages locaux et les pratiques spatiales des familles.

Dans ce contexte difficile, les Roms ne sont pas perçus comme des immigrés en situation précaire mais comme un groupe avec des pratiques particulières, inadaptées et qui nécessite leur responsabilisation. Une sorte de «mieux vaut prévenir que guérir » directement en lien avec la culture. Peut-être ne faut-il pas ignorer des éléments culturels mais l'approche culturaliste ignore de fait les raisons du non-recours, ici aux soins, et comme l'exprime l'auteure «il n'est pas rare que les soignants attribuent ces comportements à des attitudes irresponsables et ne perçoivent pas le caractère systémique et le poids du système de santé lui-même dans le non-recours aux soins » (Nacu,2009).

Pour conclure ce chapitre sur la dépendance des familles à des acteurs qui les aident, soulignons tout de même que ce serait se tromper que d'estimer que ces familles ont une totale dépendance envers les institutions ou les associations qui les soutiennent. Il ne faut pas ignorer les capacités des individus à diriger leur projet, leur vie tout en sachant quelle institution, quelle association (ou aucune d'elle) pourra être mobilisée pour y parvenir. C'est ce que souligne Martin Olivera (Olivera,2013,p8) :

«Ceux que l'on présente souvent comme des « cas sociaux transhistoriques » ne manquent en effet pas de ressources personnelles et collectives et sont loin de vivre dans l'anomie. Ils pourraient même être qualifiés de « professionnels de l'insertion locale " si l'on mesure leur capacité à maintenir leur présence dans des territoires généralement hostiles tout en entretenant une riche vie sociale et familiale. » 


\section{La migration des Roms érigée comme « problème public »}

\section{La construction de la « question rom »}

Aujourd'hui incontournable, cette «question rom » que certains politiques n'hésitent pas à appeler «problème rom » fut d'abord mise en évidence par quelques activistes roms euxmêmes. En effet, quand chutaient un à un les régimes communistes à l'Est de l'Europe, quelques associations roms se sont constituées autour de leaders qui ont interpellé les institutions européennes sur le sort peu enviable des Roms de l'Est de l'Europe. Ces institutions se sont rapidement emparées du sujet dans des objectifs d'intégration, d'insertion, et aujourd'hui d'inclusion de ces populations pour reprendre l'évolution de la terminologie en vingt ans et de projets en faveur des Roms et de lutte contre les discriminations à leur égard. Aujourd'hui l'évidence d'un problème régler a fait son chemin et elle permet d'alimenter, dans un cerce vicieux, des politiques de rejets auxquelles viennent se confronter celle de la prise en charge à tout prix. Ainsi plus elle mise en évidence comme une urgence sociale et politique plus se développe sa récupération politique pour justifier du rejet d'un à un mode de vie qui poserait problème. Politiques de la prise en charge ou des bonnes pratiques et politiques de l'exclusion et du rejet s'opposent mais reposent finalement sur les même fondements : ceux d'une question à régler, d'un problème à solutionner.

La situation des Roms aujourd'hui en Europe ne prête donc pas à l'optimisme. Elle est indissociable du contexte européen. Combien de fois a-t-on entendu qu'il s'agissait là d'une «question européenne ». La remise en cause d'une fabrique dangereuse a peu d'effet et la « question rom » ouvre alors la voir aux populismes.

\section{De la « question rom » à la montée des populismes : inquiétudes}

Comme nous l'avons dit, le socle que constitue la «question rom » permet aux politiques volontaristes ou dites des bonnes pratiques et à celles du rejet des Roms d'évoluer en parallèle à partir de l'idée de base qu'il existe un problème à régler. Ainsi, et de ce point de vue, la façon de penser les Roms dans les sociétés européennes aujourd'hui est assez semblable à celle de l'entre-deux guerres à l'égard des Tsiganes ou des nomades. 
Les nombreux programmes européens en faveur des Roms comme la décennie de «l'inclusion des Roms»(2005-2015) ont pour objectif de les «inclure» dans tous les domaines de la vie: emploi, santé, scolarisation, logement et de lutter contre les discriminations. Dans une démarche tout à fait opposée au premier abord mais qui repose sur les mêmes leviers, les «anti-tsiganes » font valoir le danger représenté par une minorité unie, homogène, au fort taux de natalité, auto-marginalisée, différente, criminelle...

De ce point de vue, le projet européen pour les Roms, alors qu'une grande partie de ses objectifs est de lutter contre la stigmatisation et les discriminations ce qu'il est difficile de condamner, contribue à maintenir une image des Roms ou des Tsiganes héritées des imageries folkloriques du XIXème siècle. Ces représentations n'ont que très peu évolué, c'est ce que souligne l'historienne Henriette Asséo en affirmant que «l'action européenne sollicite les Tsiganes dans une catégorie abstraite de diaspora, ce qui prolonge la vision des Tsiganes comme nomades qui se jouent des frontières ».

Ces deux visions, l'une inclusive et l'autre excluante, ne luttent pas vraiment l'une contre l'autre, elles alimentent la même idéologie globalisante et stigmatisante. Plusieurs mesures politiques (sans parler des exactions violentes commises par des extrémistes) «anti-roms » ont de quoi inquiéter en Europe. Citons le fichage des Roms en Italie, le discours de Grenoble de Nicolas Sarkozy en 2010, l'absence de volonté d'intégration des Roms dénoncé par Manuel Valls en 2013 alors ministre de l'intérieur ou encore le programme politique du Fidesz en Hongrie et de son premier ministre Viktor Orban sont autant de prise de positions politiques qui peuvent inquiéter.

Dans un contexte de crise économique, les angoisses se cristallisent notamment autour de quelques boucs émissaires en Europe comme les musulmans et les Roms. La population majoritaire est interpellée à ce sujet et il est de la responsabilité des politiques de ne pas instrumentaliser le phénomène. Le philosophe Patrice Maniglier l'exprime ainsi : « décidément non il n'y a pas de question rom. Il y a une question populiste adressée à la population française, à nous tous, et une question morale à propos de l'action politique » (Maniglier, 2014,p139). 
Aujourd'hui la reconnaissance des Roms, de leur culture, de leur différence n'est peut-être plus l'enjeu premier dans un tel contexte. En effet l'exacerbation de la différence stimule le populisme et le rejet et mieux vaudrait ne plus considérer les Roms comme des citoyens à part et différents mais comme d'historiques européens et nationaux qui devraient accéder au droit commun plutôt qu'à un statut de minorité ethno-culturelle qui les marginalise. 


\section{Bibliographie}

Asséo, Henriette. L'avènement politique des Roms (Tsiganes) et le génocide. La construction mémorielle en Allemagne et en France. Le Temps des Médias, Nouveau Monde Editions,2005, 5, 78-91

Clanet dit Lamanit, Elisabeth. L'objet origine et le concept d'un peuple dit « rom ». Lignes. $\mathrm{N}^{\circ} 34.2011$.

Cousin, Grégoire. Rroms migrants. Tsiganes Hier, Rroms migrants aujourd'hui ?, EspacesTemps.net, Dans l'air.

Delépine , Samuel. Atlas des Tsiganes. Les dessous de la «question rom». Autrement. Paris.93p.

Delépine,,Samuel \& Veriter,A Alexia. La non scolarisation des enfants roms migrants . In VEI Diversité. 2009. №159. P 76 à 80.

Maniglier, Patrice. Il n’y a pas de question rom. Les Temps Modernes. №677. 2014. P126139.

Nacu, Alexandra. Les Roms migrants et la santé. Diversité, 2009 ; 159 : pp. 49-55.

Olivera, Martin. Insertion ou bricolage ?2013. In Plein Droit n 99 . GISTI. P7 à 10. 